\title{
Identifying effective management factors across human errors - A case in elevator installation
}

\author{
Pin-Chao Liao; Zhangming Ma; Heap-Yih Chong
}

\begin{abstract}
Human errors are recognized as the main factor in causing construction accidents. Previous studies mainly focused on justifying theories associated with human errors and hardly quantifying the causal relations between external stimuli and human errors. Hence, the aim of this research is to develop comprehensive management measurements for addressing human errors in construction projects. Deductive reasoning was used to describe the methodological process. Firstly, we constructed a theoretical model of human errors based on Cognitive Reliability Error Analysis Method (CREAM) and knowledge-combined structure learning algorithm. Then, Bootstrap method was adopted to verify the reliability of network topology, while the similarity-flooding algorithm was used to analyze similarity of factors across various causal models for their commonalities. Subsequently, Bayesian parameter estimation was to analyze the sensitivity of the nodes. The results show that inadequate quality control, design failure and inattention are the most fundamental causes of human errors in promoting safety management. This research has proposed an analytical approach that consolidated the influential mechanics to reflect the overall influence of a root cause in the human error. Ultimately, the research lays an analytical foundation for safety management research in the future.
\end{abstract}

Keywords: human error, CREAM, Bayesian network, human factor, construction industry

\section{Introduction}

Construction industry is one of the most hazardous industries, where human errors directly lead to construction accidents (Hayhurst, 1959). Human errors can be defined as dangerous practices that violate the 
labor discipline, procedures or methods in the course of professional activities (Abdelhamid \& Everett, 2000).

An approximate of 70\%-90\% accidents has a direct or an indirect relationship with human errors (Li et al.,

2012). Although many safety management policies have been implemented, the search for an effective

management in relation to human errors is still ongoing.

Previous studies had identified the factors in affecting human errors, which were mainly related to the working environments (Akyuz \& Celik, 2015). In particular, (Cupido, 2009) built a model to describe the relationship between work behavior and work demand, which the chosen of unsafe behavior was because of workers' deficient ability to meet the work demand rather than lack of safety knowledge. The safety accident in the construction industry is usually caused by the interaction of human and environment and the environment may directly lead to human errors (Fang et al., 2016). However, very limited studies focus on the mechanism of the causal relation between external stimuli and various types of human errors.

Therefore, the research aims to develop comprehensive management measurements for addressing human errors in construction projects. An elevator installation was selected as the scope of the research due to falls from heights as the common single-fatality incidents, such as accounting for about $40 \%$ (364 out of 937 cases) of all fatalities in United States of America (Bureau of Labor Statistics, 2015). Moreover, elevator installation is one of the work at heights accidents that leads to falls (Pierce B, 2016). Deductive reasoning was adopted in designing the four research steps in the research, namely,

- Step 1: Construct the actual human error model based on Cognitive Reliability Error Analysis Method (CREAM) and knowledge-combined structure learning algorithm

- Step 2: Use the Bootstrap method to verify the reliability of network topology.

- Step 3: Use the algorithm of similarity-flooding to compare the similarities and differences between different topologies. 
- Step 4: Use the Bayesian parameter estimation to analyze the sensitivity of the nodes.

\section{Literature review}

Previous related works and theories need to be examined in order to construct the casual model that would determine the relationship between the working environments and human errors. Griffin \& Neal (2000) carried out the first study in this area, which was to evaluate the influence of safety climate on safety performance. Then, Neal et al. (2000) studied the interrelationships of general organization climate, safety climate and self-report safety behavior. These studies have provided a good research foundation and synergy between the working environments and human errors. However, there is no explanation on the causal relationship of the factors. To solve the problem, the structural equation model and the Bayesian network were used in examining the safety factors. Mohammadfam and Moghimbeigi (2017) used the Bayesian network to analyze and sort nine factors based on the sensitivity on safety behavior. García-Herrero et al. (2012) included hygiene conditions, ergonomic conditions, job demands, physical symptoms and psychological symptoms in to the Bayesian model to assess the relationships between influence factors and occupational accidents. Although the related works have examined the effects of various factors on human errors, there are still three limitations that need to be addressed because (a) the influence factors on human errors were mainly based on personal experience, (b) scholars tend to see human errors as a whole and have not considered the inherent diversity of human errors, and (c) there is no quantitative validation of reliability of the proposed models.

To address the first and second limitations, the CREAM approach can be used in the modelling process (Hollnagel, 1998). It is a second-generation method of the human reliability analysis, which has been used as a basic model to provide a mechanism for the influence of the work environment on human behavior. In the structure of CREAM, human errors can be divided into eight types of errors based on the observed 
phenomena and external manifestation, such as, duration errors, timing errors, speed errors, distance errors, direction errors, force errors, object errors and sequence errors (Wang \& Shen, 2005). In order to identify the root causes of different types of human errors, the CREAM approach offers an antecedent-consequent table that contains the relationship between the human errors and antecedents. Hence, different direct causes of the human errors will be classified into the specific antecedents, which are specified in the antecedent-consequent table based on its external manifestation. As a result, when generating any behavior associated with human errors, the wrong behavior and its different levels of antecedent will be corresponded to the antecedentconsequent table.

In the conventional method of constructing causal model, the factors that influence human error have not been unified. By referring to the CREAM approach and its antecedent-consequent table, various factors can be standardized so that any actions that produces human errors can correspond to this causal model. Moreover, due to the existence of different types of human errors in the CREAM approach, the universality of the causal model has also been conducted on accident analysis and risk assessment. Akyuz \& Celik, (2015) used the CREAM approach to analyze the operation process of the marine transportation and predict the probability of failure of operators in loading and unloading goods. In addition, Liao et al. (2012) identified the weak safety awareness and inadequate supervision were the causes of school bus accidents using the CREAM approach. Skalle et al. (2014) also proved the feasibility of CREAM in the analysis of human errors in marine traffic accidents.

Apart from that, when building a causal model with a Bayesian network, the advantage is the practical industry data can be introduced into the model for probability calculation, but the limitation is the difficulty to construct a unified model. The proposition of the CREAM has combined with many expert knowledge. As a result, through the integration of CREAM and Bayesian network, the basis of the model from expert 
experience and the actual industry data can be integrated into one model to overcome their own shortcomings.

Besides, almost all Bayesian models can analyze the sensitivity of the nodes, but the reliability of the model has not been well-verified. For example, Mohammadfam et al. (2017) analyzed the sensitivity of management commitment, supporting environment, employees participation and other six factors on safety behaviors but the model has not been verified for its reliability. Liao et al. (2016) developed a Bayesian network to identify how the design had led to unsafe behavior and calculated the probability of the unsafe behavior, but its model has not considered the verification process comprehensively. Furthermore, most safety data are rather unique and limited. The method of bootstrapping can be applied to address this issue by converting the small sample-size problem into a large-size sample-size (Streukens \& Leroi-Werelds, 2016). It is a popular nonparametric statistical method in the field of reliability verification in recent years. Li et al. (2015) used the bootstrapping approach to characterize the uncertainties in parameters and types of the bestfit marginal distributions and copulas. It indicated that the bootstrap method could effectively characterize the uncertainty in probabilistic models that derived from a small sample. Rodrigues \& Silva, (2013) combined the bootstrap technique with calibration models to determine the confidence intervals of power distribution equipment and successfully estimated the bounds for the reliability data at a specified significance level. Othman \& Musirin(2011) used the bootstrap sample in the determination of transmission reliability margin at every time interval. Basically, the idea of bootstrap is rather simple and has a profound impact on the statistical theories or towards some traditional problems (Xie and Zhu, 2008). Hence, the bootstrapping approach has several advantages, particularly to obtain the conditional probability values and verify the reliability of the topological structure as required in this research.

Last but not least, there are four types of human errors based on the theory of CREAM, which the casual relationship model will have more than one topology. It will then create a huge issue in analyzing and 
comparing these structures. Therefore, the algorithm of graph similarity-flooding can be used to address this issue as the function of the algorithm will consider two graphs as input and produce a mapping between corresponding nodes of the graphs as output (Melnik et al., 2002).

\section{Methodology}

\subsection{Constructing topology by knowledge-combined Bayesian structural learning}

\section{algorithm}

\subsubsection{Constructing the theoretical model}

The first step of this research is to construct a theoretical topological model of human errors using the CREAM approach. In order to use this approach effectively in constructing the causal model, Shen et al. (2005) have collated and simplified the variables of its original method and improved the causal relation. On this theoretical foundation, this research would construct the theoretical model of human errors by arranging the node variables and the causal relationship between variables. All the variables and their meanings were referred from the previous study (Shen et al., 2005). Table 1 shows part of the model variables and their associated terms. By referring $\mathrm{K} 1$ as an example, the meaning of the node is maintenance failure that includes an abnormal function of indicator and equipment because of lack of quality control and management. Moreover, $\mathrm{K} 1$ is a root node which leading to the human error as per the CREAM approach.

Table 1. The variables in the model

\begin{tabular}{cccc}
\hline Variable & Terms & Variable & Terms \\
\hline K1 & Maintenance failure & D4 & Inattention \\
K2 & Inadequate quality control & J1 & Communication failure \\
K3 & Management problem & N2 & Inadequate work place \\
K4 & Design failure & A1 & Observation missing \\
\hline
\end{tabular}




\begin{tabular}{cccc}
\hline M1 & Improper environment & J2 & Missing information \\
L2 & Insufficient knowledge & B1 & Faulty diagnosis \\
H1 & Operation limitation & D1 & Memory failure \\
G1 & Inadequate procedure & X1 & Human errors \\
\hline
\end{tabular}

Besides, human errors (X1) were divided into eight types of errors with different direct antecedents. In order to facilitate the construction of theoretical model and the mapping from actual project data to node variables, we merged human errors into four categories according to their description, such as, wrong sequence (including sequence errors), wrong action (including force errors, speed errors, distance errors and direction errors), wrong target (including object errors) and wrong time (including duration errors and timing errors). After merging different human error and integrating their possible antecedents, the possible causes of four types of human error are shown in Table 2.

Table 2. The antecedents of the human errors

\begin{tabular}{ll}
\hline Types of human errors & General antecedents \\
\hline wrong sequence & A2,B1,C1,D1,D4,G1,H1,J1 \\
wrong action & A1,B1,C1,D2,D3,D4,F1,G1,H2,I2,J1 \\
wrong target & A1,A2,C2,D3,D4,G1,I1,J1 \\
wrong time & A1,A2,B1,B4,C1,D4,G1,J1 \\
\hline
\end{tabular}

For example, the "wrong sequence" has eight antecedents, respectively missed observation (A2), failed diagnosis (B1), inappropriate plan (C1), memory error (D1), inattention (D4), imperfect procedures (G1), limited operation (H1) and failed communication (G1). We can get the general antecedents of other three types of human errors that directly affect X1 like wrong sequence, and then work backwards to get the 
antecedents of these nodes layer by layer until there is no antecedent for a variable according to the antecedent-consequent relationship of human error. This refers to the specific backtracking process (Shen, et al., 2005). Then, he topology would be formed, which the antecedents represented the parent node of the specific node and the causal relationship was based on the chains of antecedent-consequent table. However, due to the existence of cycle relationship in the antecedent-consequent table, some loops in the final theoretical topology has conflicted with the definition of Bayesian network. In order to eliminate these cycles, the model deleted the causal relationship that did not comply with the law of the risk and had a low occurrence probability as per the actual situation in the elevator installation. To ensure the completeness of the topology, the final model was reviewed and validated by an expert panel comprised of eight experienced installation workers (aged 31-42 years old, with 14-22 years of working experience and high-school education backgrounds), six safety inspectors (aged 29-42 years old, with 5-18 years of work experience and undergraduate education backgrounds) and four foremen (aged 29-38 years old, with 10-16 years of working experience and high-school education backgrounds).

As a result, four different theoretical models were developed based on the four types of human errors and their antecedents. The four models then would serve as the foundation for the subsequent analysis.

\subsubsection{Data sources and mapping methods}

Although the CREAM approach has been widely used to predict human errors, the authenticity and reliability of the model still need to be carefully examined as to address the lack of practical and supporting industry data. In order to solve the problem, the actual industry data needs to be obtained first. Then, the mapping can be conducted and compared between the industry data and theoretical models.

In the field of elevator installation, the daily safety inspection has been considered as a core part of the site management. Each company would use a safety checklist to detect any possible safety risks in the 
workplace. Fatality Prevention Audit (FPA) is the first proposed safety checklist among them. It has become the template for various companies to use at this moment. In this research, the safety inspection data of the selected elevator company were collected for the years of 2010-2015. The FPA checklist was classified into four parts by letter, namely, A for fall protection, B for elevator/escalator control, C for hazardous energy control and D for high-risk operation. It contained 81 items that covered a comprehensive site situation of the project.

During the process of safety inspection, each item of the FPA checklist was carefully checked to form a check record and each record formed the data for calculation. We stipulated that the value of a specific FPA item as 1 when the item would occur, otherwise the item would be recorded as 0 . Such similar safety inspection would be repeated many times. As a result, 39691 data sets were collected.

In order to establish mapping between the FPA data and theoretical models, the research adopted a method of semantic resolution to find the match between the items in the FPA and the variable nodes in the models. Firstly, the description of CREAM's variable nodes and the FPA items were analyzed semantically and were compared with each other to determine the mapping relation. Moreover, we also interviewed with the experts to confirm the specific mapping. Therefore, the mapping process actually incorporated with the expert experience. Secondly, the result of mapping was modified and improved to obtain the final mapping result by checking with company. As for each node data in the theoretical model, there were one or more FPA items corresponding to it. In addition, some nodes were treated as null nodes due to different structure between FPA and CREAM. Table 3 shows the partial mapping relations.

Table 3. Items in FPA and its description

Variable Item Description


C06 Mechanics are working on de-energized equipment that have not been locked \& tagged (mainline, signal, fan \& light circuit, etc.).

C12 Employees are working in wet pit with power on.

C13 Jewelry and other metallic objects worn around live equipment.

B1

C1

D1

D4

F1

A02 Guardrails are not adequate and no fall protection used.

C08 Use of metal ladders where there is a potential for contact with electrical circuits.

A06 More than one person tied off to the same lifeline.

B04 More than two people working in the hoist-way without proper authorization.

C09 Failure to use Ground Fault Circuit Interrupters or equivalent protective devices (rated at 10mA max. trip level or less).

A09 Improper sequence of connecting and disconnecting lanyard.

B01 Improper verification of safety chain function (door, E-Stop) and Inspection Switch.

B07 Improper verification of safety chain function (door, E-Stop).

D05 Knots tied in slings.

D08 Working under suspended load.

A03 Fall protection equipment not certified or does not conform to Otis requirements.

A07 Lifelines not protected from sharp edges.

B09 Improper door blocking device.

B1 TO5 located too far from landing and no alternate safe procedure used.

B08 Stop switch located too far from landing and no alternate safe procedure used.

B12 Mechanism (switches, ladder, releases, etc.) locations prevent use of standard procedure. No alternative safe procedure available or used. 

locate this info.

A08 Inadequate or unknown capacity of anchorage point for lifeline and/or lanyard.

D10 No certificates or inspection records (inability to assure that scaffold conforms to recognized standards).

D01 Out-of-date or no inspection of lifting apparatus.

D20 Failure to inspect and maintain false car in good working condition.

A10 Ladder not secured at elevations greater than 2 meters.

B02 No Top of Car Inspection installed.

K3 C05 Multiple employees have keys/combinations to all locks.

D07 Damaged slings not removed from service.

D22 False car erected by untrained personnel w/o use of instructional guidelines.

K4 B05 TOCI located too far from landing and no alternate safe procedure used.

B12 Mechanism (switches, ladder, releases, etc.) locations prevent use of standard procedure. No alternative safe procedure available or used.

D21 Improper activation, construction and functioning of safeties.

L2 C25 Unsafe oxygen-acetylene or compressed gases welding, cutting, heating equipment or procedures.

D25 Employees are not familiar with false car construction requirements.

D30 Mechanics could not explain use of jumpers.

M1 C07 Inadequate electrical protection in proximity of work activity. 
D07 Damaged slings are not removed from service.

D29 Jumpers are in place when mechanic departed jobsite.

X1:wrong A05 No fall protection while working on ladder at elevations greater than 2 meters.

sequence A02 Guardrails are not adequate and no fall protection used.

C10 Mechanic did not verify a "zero energy state" (power, signal, fan \& lights).

X1:wrong B03 Riding the car top in Normal operation.

action $\quad$ C22 Working in close proximity to unguarded drive or diverter sheaves or other rotating equipment.

D10 No certificates or inspection records (inability to assure that scaffold conforms to recognized standards).

X1:wrong A11 Riding car top with long lanyard w/o inspection mode by 2 independent means.

target $\quad$ C14 Use of uninsulated tools around live equipment.

D27 Use of unauthorized jumpers (on site, on person, in tool box, etc.).

\subsubsection{Constructing the actual model by Bayesian structural learning}

Bayesian structural learning approach is a powerful and flexible tool to mine and identify the dependencies among nodes, and then to form the network topology. Since the topology was constructed based on the CREAM approach and there was no actual data input, the problem of construction human-error model based on experience was not solved yet. Therefore, after completing of the data mapping, we adopted the method of Bayesian structural learning to construct an improved network topology combining prior knowledge and real data. 
There are two most common Bayesian structural learning algorithms: the algorithm of searching the best model based on the maximum score, and the algorithm based on the relationship between nodes. By comparing the algorithm characteristics, we utilized the algorithm based on the relationship between nodes and combined with prior knowledge in this research to improve the network topology under the five processes.

Step 1: Initialization of the model. For the node $X_{i}: X_{i} \in A, \mathrm{i}=1,2, \ldots \mathrm{n}$ (A is a set of all the nodes in the model, a total of $\mathrm{N}$ nodes), the potential parent nodes set is denoted as $P_{i}$.

Step 2: Preprocessing of the model. For the node $X_{i}$, use chi-square test and fisher's exact test to check whether the nodes before $X_{i}$ are independent with $X_{i}$. Put all the nodes that are independent with the $X_{i}$ into a set of impossible parent nodes, denoted as $N P_{i}\left(\right.$ not $P_{i}$ ). At the same time, if there is an independence node in $P_{i}$, the node is removed from the $P_{i}$.

Step3: Checking of the correctness of the parent node set. If there is a node $X_{j}: X_{j} \notin P_{i}$ and $X_{j} \notin N P_{i}$, give a set of nodes in $P_{i}$ to test the conditional independence between $X_{j}$ and $X_{j}$. If $X_{j}$ and $X_{j}$ are not conditional independence, $P_{i}$ need to be adjusted due to wrong judgment from prior knowledge. The method of adjustment is to put all nodes that are before $X_{i}$ and not in $N P_{i}$ into $P_{i}$ as the new $P_{i}$.

Step 4: For the newly generated $P_{i}$ in step 2, the conditional independence test is performed to determine and delete the set of possible parent nodes. If $X_{j}: X_{j} \in P_{i}$ exists, the remaining nodes in $P_{i}$ is given, if $X_{j}$ and $X_{i}$ are conditional independence, delete $X_{j}$ from $P_{i}$. For newly formed $P_{i}^{\prime}$, repeat the above steps until there is no $X_{j}$. Then all nodes in the existing $P_{i}^{\prime}$ are the parent nodes of $X_{i}$ and connection is established between parent nodes and child node.

Step 5: The Step 2, Step 3, and Step 4 steps are repeated to the next node $X_{i+1}$ according to the node order until all the nodes in the model are checked and finally the structure learning model is determined.

To sum up, we set up a maximum range of potential parent nodes according to the prior knowledge and 
the preliminary independence test. And then, some variables were removed according to the conditional probability test. This algorithm could reduce the amount of computation, but also could avoid the errors that were completely dependent on the prior knowledge. The algorithm was actualized by JAVA in Eclipse software.

\subsection{Verifying robustness by bootstrap}

After constructing the topology, the reliability of the causal relationship between parent node and child node needs to be verified. Hence, the Bootstrap method was adopted for the verification process, which mainly considered for the significance of the influence from parent nodes' change to child nodes.

By using the bootstrapping method, multiple data sets were acquired to get new data sets and calculated the desired statistic repeatedly which could be used in the significance test of the model. The following steps were conducted for testing the robustness of the model by using Bootstrap Method:

- Step 1: It was to determine the original data set. The origin data was collected from the FPA checklist of an elevator installation company with a total of 39000 on-site observations. Each data contains the information whether the item happens or not. If there is an item that the variable node in topology contains happens, then the node is recorded as 1 , otherwise the node is recorded as 0 .

- Step 2: It was to determine the size of the sample and obtain a new dataset. According to the description of the bootstrap method, we acquire 39691 data for each variable by simple random sampling with replacement. Taking X1 as an example, there are 39691 observations for X1 and we randomly pick one observation at a time with replacement and after repeating for 39691 times, we obtained a new dataset. In the original data which was be extracted, some observation would be selected for more than one time and some observation wouldn't be selected even once. As a result, a new data set was generated.

- Step 3: It was to determine the number of new data sets required. The number of new data sets 
depends on the time of step 2. In consideration of the more times we repeat, the less chance in the process of simulation, we repeated the step 2 for 5000 times and obtained 5000 new data sets.

- Step 4: It was to repeat parameter estimation based on the 5000 new data sets from step 3. In the process of Bayesian parameter learning, the data was used all at once and conditional probability table was calculated. Now that we had 5000 data sets, we can estimate parameters in the Bayesian framework for 5000 times and get 5000 different conditional probability tables that represents the probability that a child node will occur in different conditions of its parent node.

- Step 5: It was to determine the rules of significance test. In the topology, different states of parent node have different child node probabilities, so we need to set the rules for comparing these different probabilities. The rules are as follows: when verifying the significant effect of a specific parent node on its child node, set the state of the other parent nodes not occur at first, and then compare whether there is significant difference between the probabilities of child nodes' occurrence in two states of the specific parent node. Considering the value of each node in topology is 1 or 0 which represents happen or not, the rules are simplified as follows: child node equals to 1 , other parent nodes equals to 0 , compare the probability difference between the specific parent node equals to 1 and equals to 0 .

- Step 6: It was to verify the robustness of the model based on the rules as proposed in step 5. In step 4 and step 5, 5000 different results of parameter estimation were obtained, so in step 6 we were able to test the significant difference. Due to the unknown and unclear of the variance in the two sets of data, the functionality of $\mathrm{T}$ test was applied to analyze the result of significant test for each parent-child node pair. 


\subsection{Similarity-flooding algorithm}

Consequently, the differences between the topologies and the similarity of corresponding nodes in structure were compared (Melnik et al., 2002). It used two graphs as input and output for the mapping between corresponding nodes of the graphs. More specifically, the similarity of correspond nodes was calculated after inputting the nodes and edges of topologies into the algorithm. According to the similarityflooding algorithm of the open source program in Perl, we added the nodes and edges from different models of human errors into the program and set the number of iteration to 500 times to get the stable comparison results.

\subsection{Sensitivity analysis based on Bayesian parameter estimation.}

After building the Bayesian network, the conditional probability table (CPT) for each variable was calculated using Bayesian parameter estimation. Each cell in a CPT was called as a parameter. ExpectationMaximization (EM) algorithm was used. The algorithm alternated between the expectation step and the maximization step. In the expectation step, the missing data were filled in and in the maximization step, which the filled data set was used to calculate a new maximum likelihood estimation for each parameter. The Bayesian network provided the function of parameter estimation based on EM algorithm using the Matlab software.

The process of sensitivity analysis reflected the effect of the change of variable on another variable. According to the definition of sensitivity analysis, the value of each cell was changed and calculated based on the conditional probability of human errors in the Matlab software. After comparing the conditional probability of human errors between two states of antecedent nodes, the differences of two values were reflected the sensitivity of human error to antecedent node. 


\section{Result}

\subsection{The result of the constructed topology}

The CREAM approach was adopted successfully and demonstrated a causal relationship between nodes

and nodes for the causal model of human errors. These causal relationships constituted the causal network of model as the theoretical model of this research. After mapping the data of FPA to model nodes, the CREAM theory model had the actual data of elevator installation project and thus solved the reliability issues of the model's data.

In the construction of Bayesian network, the research proposed a structure learning algorithm based on dependency. The CREAM model and the process of data mapping provided the possibility of using the algorithm. The relation was added between parent nodes and child nodes in the theoretical model, then chisquare test and conditional independence test were used to confirm the dependence and get the final network structure. Although there were four types of human errors, only three of them (wrong sequence, wrong action, wrong target) had the actual corresponding the FPA data. So, only the three models were supported by the actual industry data.

Figure 1 illustrates the three different topologies with some obvious information about nodes. For the ease of identification, $\mathrm{A}$ is the model of wrong sequence, $\mathrm{B}$ is the model of wrong action and $\mathrm{C}$ is the model of wrong target. The models have 11, 11, and 9 nodes; and 22, 27, and 16 edges respectively. Thorough the preliminary analysis of the three networks, the root nodes of the three graphs are similar. It shows the initial causation for different human errors is consistent. The impacts of K1 (maintenance failure), K2 (inadequate quality control), K3 (management problem), and K4 (design failure) have transmitted through different immediate nodes that eventually lead to the occurrence of different human errors. Therefore, maintenance failure, inadequate quality control, management problem and design failure are the original cause of human 
errors after combining the CREAM theory and practical data from the elevator company. In addition, the X1 nodes would not be the same impact in different topologies. We should pay more attention to the similarities and differences of topologies in further analysis of human errors causes.

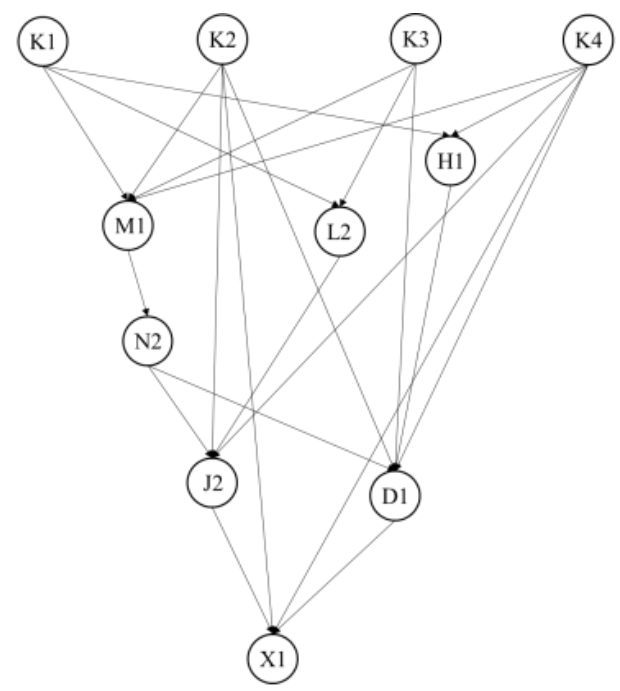

Fig. 1(a) The causal model of wrong sequence

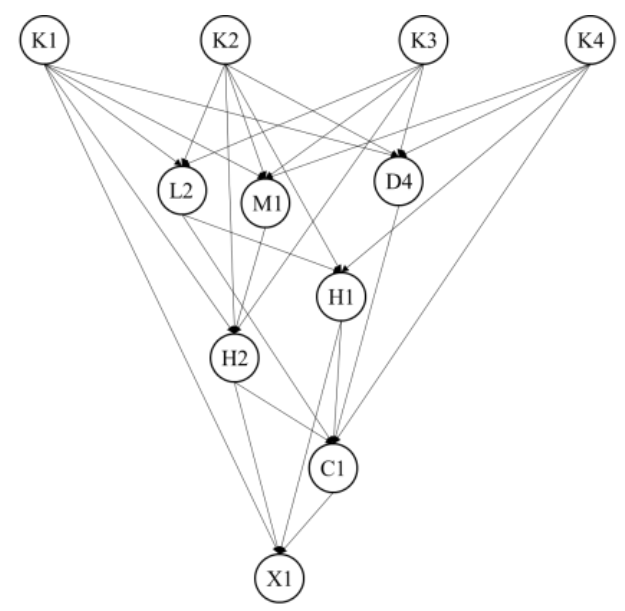

Fig. 1(b) The causal model of wrong action 


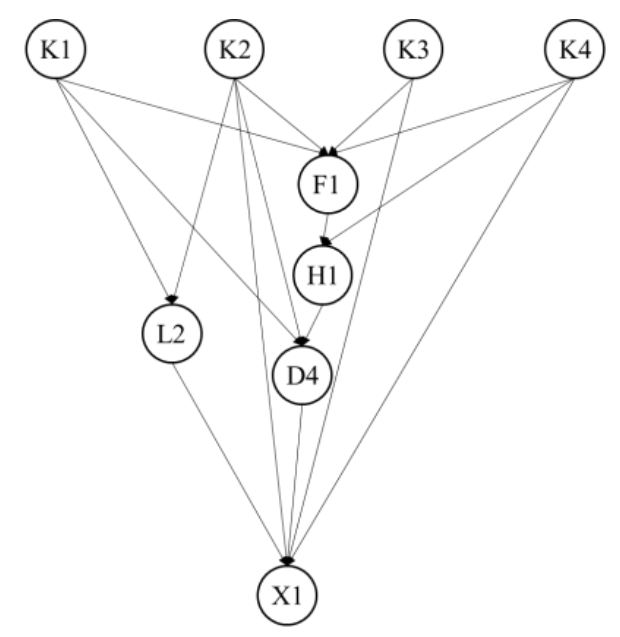

Fig. 1(c) The causal model of wrong target

\subsection{The result of reliability test}

After constructing the three models, the next step is to investigate how strong the relationship between parent nodes and child nodes. The verification method of the model's robustness is to check whether each parent node has a significance impact on its own child node by hypothesis test. If the result of the hypothesis test is significant, it means all the parent nodes has a great influence on child nodes. Then, the robustness of the model is trustworthy. Table 4 shows the results of the verification.

Table 4(a). The verification result of wrong sequence

\begin{tabular}{|c|c|c|c|c|c|c|c|c|}
\hline $\begin{array}{l}\text { child } \\
\text { node }\end{array}$ & $\begin{array}{l}\text { parent } \\
\text { node }\end{array}$ & $\mathrm{t}$-value & $\begin{array}{l}\text { child } \\
\text { node }\end{array}$ & $\begin{array}{l}\text { parent } \\
\text { node }\end{array}$ & t-value & $\begin{array}{l}\text { child } \\
\text { node }\end{array}$ & $\begin{array}{l}\text { parent } \\
\text { node }\end{array}$ & t-value \\
\hline \multirow[t]{2}{*}{$\mathrm{X} 1$} & $\mathrm{~J} 2$ & 69.99 & \multirow[t]{5}{*}{ D1 } & K4 & 651.31 & \multirow[t]{2}{*}{ H1 } & $\mathrm{K} 1$ & 352.82 \\
\hline & D1 & 28.01 & & H1 & 68.95 & & K4 & 352.83 \\
\hline \multirow[t]{3}{*}{$\mathrm{J} 2$} & N2 & 13.39 & & K2 & 229.39 & \multirow[t]{3}{*}{ M1 } & K1 & 62.34 \\
\hline & L2 & 307.33 & & $\mathrm{H} 3$ & 41.18 & & $\mathrm{~K} 2$ & 203.58 \\
\hline & K4 & 307.33 & & $\mathrm{~N} 2$ & 186.25 & & K3 & 141.68 \\
\hline
\end{tabular}




\begin{tabular}{l|l|l|l|l|l|l|l|l}
\hline \multirow{2}{*}{ 22 } & K1 & 10.90 & N2 & M1 & 262.51 & & K4 & 51.60 \\
\cline { 2 - 7 } & K3 & 22.59 & \multicolumn{5}{|c}{$t_{\alpha} \approx 1.960$} \\
\hline
\end{tabular}

Table 4(b). The verification result of wrong action

\begin{tabular}{|c|c|c|c|c|c|c|c|c|}
\hline $\begin{array}{l}\text { child } \\
\text { node }\end{array}$ & $\begin{array}{l}\text { parent } \\
\text { node }\end{array}$ & $\mathrm{t}$-value & $\begin{array}{l}\text { child } \\
\text { node }\end{array}$ & $\begin{array}{l}\text { parent } \\
\text { node }\end{array}$ & t-value & $\begin{array}{l}\text { child } \\
\text { node }\end{array}$ & $\begin{array}{l}\text { parent } \\
\text { node }\end{array}$ & t-value \\
\hline \multirow[t]{4}{*}{$\mathrm{X} 1$} & K1 & 20.21 & \multirow[t]{3}{*}{$\mathrm{H} 1$} & L2 & 215.62 & $\mathrm{H} 2$ & K3 & 8.49 \\
\hline & $\mathrm{H} 2$ & 5.94 & & $\mathrm{~K} 2$ & 73.02 & \multirow[t]{4}{*}{ D4 } & $\mathrm{K} 1$ & 5.48 \\
\hline & H1 & 40.86 & & K4 & 215.62 & & $\mathrm{~K} 2$ & 143.00 \\
\hline & $\mathrm{C} 1$ & 139.63 & L2 & $\mathrm{K} 1$ & 12.70 & & K3 & 89.79 \\
\hline \multirow[t]{5}{*}{$\mathrm{C} 1$} & $\mathrm{H} 2$ & 67.15 & & $\mathrm{~K} 2$ & 106.15 & & K4 & 10.94 \\
\hline & L2 & 179.93 & & $\mathrm{~K} 3$ & 14.21 & \multirow[t]{4}{*}{ M1 } & $\mathrm{K} 1$ & 90.32 \\
\hline & H1 & 17.06 & \multirow[t]{3}{*}{$\mathrm{H} 2$} & $\mathrm{~K} 1$ & 153.29 & & $\mathrm{~K} 2$ & 276.22 \\
\hline & D4 & 169.70 & & $\mathrm{~K} 2$ & 101.47 & & K3 & 38.09 \\
\hline & K4 & 61.88 & & M1 & 153.29 & & K4 & 597.32 \\
\hline & & & & $t_{\alpha} \approx$ & & & & \\
\hline
\end{tabular}

Table 4(c). The verification result of wrong target

\begin{tabular}{l|l|l|l|l|l|l|l|l}
\hline child & parent & & child & parent & & child & parent & t-value \\
node & node & t-value & node & node & t-value & node & node & \\
\hline X1 & L2 & 43.93 & L2 & K1 & 7.80 & H1 & F1 & 55.89 \\
\hline
\end{tabular}




\begin{tabular}{|c|c|c|c|c|c|c|c|}
\hline K2 & 505.67 & & $\mathrm{~K} 2$ & 100.93 & & K4 & 187.03 \\
\hline D4 & 809.18 & \multirow[t]{3}{*}{ D4 } & K1 & 162.32 & \multirow[t]{4}{*}{$\mathrm{F} 1$} & $\mathrm{~K} 1$ & 108.75 \\
\hline K3 & 113.59 & & $\mathrm{~K} 2$ & 100.78 & & $\mathrm{~K} 2$ & 351.66 \\
\hline K4 & 809.18 & & $\mathrm{H} 1$ & 162.32 & & K3 & 193.01 \\
\hline \multicolumn{5}{|c|}{$t_{\alpha} \approx 1.960$} & & $\mathrm{~K} 4$ & 81.64 \\
\hline
\end{tabular}

Table 4 consists of three parts: the child node, its parent nodes and the result of the hypothesis test for the effect on the child node. As all t-value is greater than $t_{\alpha}$, the child node and parent node are relative. Take $\mathrm{X} 1$ as an example, this node has two parent nodes: $\mathrm{J} 2$ and D1. The t-value of $\mathrm{J} 2$ and D1 both are greater than $t_{\alpha}$ which means $\mathrm{J} 2$ and $\mathrm{D} 1$ both have a significant impact on the occurrence of $\mathrm{X} 1$. All the t-values in the table are far greater than $t_{\alpha}$ which explain that all parent nodes have significant effects on the child nodes. It proves that the relation of each node in the topology is reliable. We can also compare the effects of two parent nodes on the same child node. The t-value of J2 is larger than D1's, which means the influence of $\mathrm{J} 2$ on $\mathrm{X} 1$ is greater than D1. In other words, loss or error of information is more likely to lead to an unsafe behavior by comparing to the memory errors. Therefore, apart from significant judgment, the effect of parent nodes on child node can be ranked and analyzed.

\subsection{The similarity result of the different topologies}

In the output of the similarity-flooding algorithm, it includes not only the comparison between the same nodes as $\mathrm{J} 2$ in wrong sequence and in wrong action, but also compares between different nodes such as, $\mathrm{K} 2$ in wrong sequence and L2 in wrong action. However, it is of no practical significance when comparing the different nodes in the two topologies, so we have filtered such results and reserved the comparison result of same nodes only. Taking A (wrong sequence) and B (wrong action) as an example, we can obtain the results 
as shown in Table 5(a).

Table 5(a). The structure similarity of nodes in model a and b

\begin{tabular}{cccc}
\hline $\mathrm{a}$ (wrong sequence) & $\mathrm{b}$ (wrong action) & representative meaning & similarity \\
\hline $\mathrm{H} 1$ & $\mathrm{H} 1$ & Operation limitation & 0.31 \\
$\mathrm{~K} 1$ & $\mathrm{~K} 1$ & Maintenance failure & 0.58 \\
$\mathrm{~K} 2$ & $\mathrm{~K} 2$ & Inadequate quality control & 0.77 \\
$\mathrm{~K} 3$ & $\mathrm{~K} 3$ & Management problem & 0.46 \\
$\mathrm{~K} 4$ & $\mathrm{~K} 4$ & Design failure & 0.77 \\
$\mathrm{~L} 2$ & $\mathrm{~L} 2$ & Insufficient knowledge & 0.31 \\
$\mathrm{M} 1$ & $\mathrm{M} 1$ & Improper environment & 0.65 \\
$\mathrm{X} 1$ & $\mathrm{X} 1$ & Human error & 0.62 \\
\hline
\end{tabular}

The similarity of $\mathrm{K} 2$ and $\mathrm{K} 4$ is the highest in the comparison between the two topologies. Besides, the similarity of $\mathrm{H} 1$ is low. It indicates that the network structure of operation limitation is quite different in wrong sequence and wrong action. Similarly, we can obtain other comparative results as shown in Table 5.B and 5.C.

Table 5(b). The structure similarity of nodes in model a and c

\begin{tabular}{cccc}
\hline a(wrong sequence) & $\mathrm{c}$ (wrong target) & representative meaning & similarity \\
\hline $\mathrm{H} 1$ & $\mathrm{H} 1$ & Operation limitation & 0.24 \\
$\mathrm{~K} 1$ & $\mathrm{~K} 1$ & Maintenance failure & 0.43 \\
$\mathrm{~K} 2$ & $\mathrm{~K} 2$ & Inadequate quality control & 0.76 \\
$\mathrm{~K} 3$ & $\mathrm{~K} 3$ & Management problem & 0.29
\end{tabular}




\begin{tabular}{|c|c|c|c|}
\hline K4 & K4 & Design failure & 0.48 \\
\hline $\mathrm{L} 2$ & $\mathrm{~L} 2$ & Insufficient knowledge & 0.24 \\
\hline $\mathrm{X} 1$ & $\mathrm{X} 1$ & Human error & 0.76 \\
\hline \multicolumn{4}{|c|}{ Table 5(c). The structure similarity of nodes in model b and c } \\
\hline $\mathrm{b}$ (wrong action) & $\mathrm{c}($ wrong target $)$ & representative meaning & similarity \\
\hline D4 & D4 & Inattention & 0.62 \\
\hline H1 & $\mathrm{H} 1$ & Operation limitation & 0.38 \\
\hline K1 & K1 & Maintenance failure & 0.71 \\
\hline K2 & $\mathrm{K} 2$ & Inadequate quality control & 0.95 \\
\hline K3 & $\mathrm{K} 3$ & Management problem & 0.38 \\
\hline K4 & K4 & Design failure & 0.38 \\
\hline L2 & $\mathrm{L} 2$ & Insufficient knowledge & 0.38 \\
\hline $\mathrm{X} 1$ & $\mathrm{X} 1$ & Human error & 0.76 \\
\hline
\end{tabular}

The results show some nodes have appeared in three models, such as K1, K2, K3, K4 H1, L2 and X1; while some nodes have only appeared in two models, such as M1 for the wrong sequence and wrong action, D4 for the wrong action and wrong target. Moreover, some nodes have only appeared for one model, such as $\mathrm{N} 2, \mathrm{~J} 2, \mathrm{D} 1$ in wrong sequence, $\mathrm{H} 2, \mathrm{C} 1$ in wrong action and $\mathrm{F} 1$ in wrong target. The presence or absence of these nodes reflects the similarities and differences between the topologies. In order to further analyze the similarities between the topologies, we have integrated the similarity of the nodes as shown in Table 6 .

Table 6. Integration of comparison results of three models same node A and B A and C B and C average similarity rank 


\begin{tabular}{cccccc}
\hline K1 & 0.58 & 0.43 & 0.71 & 0.57 & 3 \\
K2 & 0.77 & 0.76 & 0.95 & 0.83 & 1 \\
K3 & 0.46 & 0.29 & 0.38 & 0.38 & 5 \\
K4 & 0.77 & 0.48 & 0.38 & 0.54 & 4 \\
M1 & 0.65 & 0 & 0 & 0.22 & 8 \\
D4 & 0 & 0 & 0.62 & 0.21 & 9 \\
H1 & 0.31 & 0.24 & 0.38 & 0.31 & 6 \\
L2 & 0.31 & 0.24 & 0.38 & 0.31 & 6 \\
X1 & 0.62 & 0.76 & 0.76 & 0.71 & 2 \\
\hline
\end{tabular}

According to the sorting result, the similarity of $\mathrm{K} 1, \mathrm{~K} 2, \mathrm{~K} 3, \mathrm{~K} 4$ and $\mathrm{X} 1$ is significantly higher than other intermediate nodes. Since K1, K2, K3, K4 are the standalone nodes of the model, there are no parent nodes; $\mathrm{X} 1$ is the only root node of the model, and there are no child nodes as observed from the topology. However, the function of standalone nodes would not lead to human errors without intermediate nodes, where M1, D4, H1 and L2 are the nodes that play the role of transmission from the standalone nodes to the root nodes. Due to the existence of parent nodes and child nodes, the similarity of intermediate nodes is lower than others nodes, such as M1, D4, H1 and L2.

The higher the similarity of the nodes, the more similar mechanism the nodes have. From the project management perspective, the control of the node can be happened in all topologies, so managers can control all types of human errors by controlling a single variable.

\subsection{The result of sensitivity analysis}

Although the similarity of same nodes in different topologies has been calculated through the algorithm 
of similarity-flooding; there is no quantitative analysis of the nodes to determine its impacts on human errors.

Hence, the CPT from Bayesian network parameter estimation was used to solve this problem. The CPT analyzed the probability of various states of a node according to a different configuration of its parent states.

Table 7 shows the CPT for X1 in the model of wrong action based on the two states of nodes: (a) occur or (b) not occur.

Table 7. The CPT of X1 in the model of wrong action

\begin{tabular}{|c|c|c|c|c|c|}
\hline \multicolumn{4}{|c|}{ parents' nodes of the human error } & \multicolumn{2}{|c|}{$\mathrm{X} 1$ : human error (wrong sequence) } \\
\hline K1 & $\mathrm{H} 2$ & $\mathrm{H} 1$ & $\mathrm{C} 1$ & 0 & 1 \\
\hline 0 & 0 & 0 & 0 & 0.9898 & 0.0102 \\
\hline 0 & 0 & 0 & 1 & 0.9718 & 0.0282 \\
\hline 0 & 0 & 1 & 0 & 1 & 0 \\
\hline 0 & 0 & 1 & 1 & 1 & $1.615 \mathrm{e}-7$ \\
\hline 0 & 1 & 0 & 0 & 0.9799 & 0.0201 \\
\hline 0 & 1 & 0 & 1 & 0.9686 & 0.0314 \\
\hline 0 & 1 & 1 & 0 & 1 & 0 \\
\hline 0 & 1 & 1 & 1 & 1 & 0 \\
\hline 1 & 0 & 0 & 0 & 0.8485 & 0.1515 \\
\hline 1 & 0 & 0 & 1 & 0.7461 & 0.2539 \\
\hline 1 & 0 & 1 & 0 & 1 & $1 \mathrm{e}-7$ \\
\hline 1 & 0 & 1 & 1 & 1 & 0 \\
\hline 1 & 1 & 0 & 0 & 0.9120 & 0.0880 \\
\hline 1 & 1 & 0 & 1 & 0.9998 & 0.0002 \\
\hline
\end{tabular}




\begin{tabular}{cccccc}
\hline 1 & 1 & 1 & 0 & 0.9992 & $7.663 \mathrm{e}-4$ \\
1 & 1 & 1 & 1 & 1 & 0 \\
\hline
\end{tabular}

For each variable, the conditional probability describes as the occurrence probability of different states of the parent node and the occurrence probability of child nodes in different states of parent nodes. For example, X1 has two states and four parent nodes, each parent node has 2 states respectively, which lead to $2^{4} \times 2=32$ probability values for X1. In the first row, when $\mathrm{K} 1, \mathrm{H} 2, \mathrm{H} 1, \mathrm{C} 1$ have not occurred, the value will be 0 , and then the probability of $\mathrm{X} 1$ 's occurrence is 0.0102 . The meaning of other rows is similar as the first row. In addition to the conditional probability between directly connected nodes, the Bayesian network can calculate the conditional probability between non-connected nodes, such as the probability of occurrence of X1 when K2 occurs. Therefore, the probability of arbitrarily node in a particular state can be calculated by giving the value of another node. For example: set the value of K2 (design failure) to 1, we can calculate the probability of human error under the premise of design failure. Similarly, in the case of assigning K4 to 0, we can calculate the opposite probability of human error. If there is no change in site objective situation which is equivalent to control the change of variable in site, we can get the magnitude of the effect of design failure on human error by comparing the results of these two calculations. In this way, the influence of other nodes on human errors can be calculated. Table 8 shows the results of the calculation.

Table 8. The influence rate of nodes on X1

\begin{tabular}{|c|c|c|c|c|c|c|c|}
\hline \multirow[b]{2}{*}{ variable } & \multicolumn{2}{|c|}{ wrong sequence } & \multicolumn{2}{|c|}{ wrong action } & \multicolumn{2}{|c|}{ wrong target } & \multirow[b]{2}{*}{ node } \\
\hline & absolute & relative & absolute & relative & absolute & relative & \\
\hline \multirow[t]{2}{*}{ node } & influence & influence & influence & influence & influence & influence & rank \\
\hline & rate & rate & rate & rate & rate & rate & \\
\hline
\end{tabular}




\begin{tabular}{cccccccc}
\hline K1 & 0.9754 & 16.1042 & 1.2024 & 80.6056 & -0.9556 & -74.1621 & 4 \\
K2 & 12.762 & 382.2539 & 0.0034 & 0.2236 & 33.3206 & 5173.4334 & 2 \\
K3 & 0.0827 & 1.3625 & 0.0013 & 0.0853 & 0.3963 & 32.8275 & 6 \\
K4 & 4.8531 & 80.2548 & 14.6805 & 1176.1538 & 0.0272 & 2.1211 & 3 \\
M1 & -0.5455 & -8.9332 & 0.7825 & 52.3872 & - & - & 7 \\
D4 & - & - & 1.2510 & 83.8641 & 1.0885 & 85.4188 & 1 \\
L2 & 2.1746 & 35.9819 & -0.0384 & -2.5635 & 0.4717 & 36.8621 & 4 \\
H1 & 0.0355 & 0.5851 & -1.5018 & -99.9971 & -0.9865 & -76.6098 & 8 \\
\hline
\end{tabular}

By referring to the shared nodes of three topologies, the absolute influence rate and relative influence rate will reflect the impact of antecedent nodes on X1 (human error), the formula is as follows.

$$
\begin{array}{cc}
\text { absolute influence rate }=100 \times\{p(X 1=1 \mid \text { node }=1)-p(X 1=1 \mid \text { node }=0)\} & \text { Eq. (1) } \\
\text { relative influence rate }=100 \times \frac{p(X 1=1 \mid \text { node }=1)-p(X 1=1 \mid \text { node }=0)}{p(X 1=1 \mid \text { node }=0)} & \text { Eq. (2) }
\end{array}
$$

In addition, the ranking of antecedent nodes' impact has been calculated on the human error by looking at the relative influence rate for variable nodes in each topology. The influence rate of the four leaf nodes is higher than that of the intermediate nodes, but D4 plays a great role in wrong action and wrong target. There is a negative influence rate in the table, which means that the occurrence of antecedent errors helps to control the human error. The reason is that it will take up most of the attention of workers when facing these prominent risks, make workers more likely to identify other risks and thus chose the safe behavior.

\subsection{Synthesis of results}


The similarity of K2 (inadequate quality control) in different topologies is the highest among all nodes, and its influence rate is only lower than D1, so $\mathrm{K} 2$ is the most effective node to control human errors. The primary task of controlling the human error is to have better control on site facilities. In this study, the inadequate quality control includes: (a) the equipment or function cannot meet the requirements and (b) the lack of required resource. Under the inadequate quality control, site workers are likely to devote most of their efforts on how to use the machine and how to get the resources they need. One of the effective methods to control the mistake is to ensure the good working condition of the equipment and adequate resources for the workers. Besides, regular inspections of machinery and equipment, timely procurement and effective inventory management would help to prevent or mitigate these quality control issues.

Furthermore, K4 (design failure) is also a valid control node. Design failure refers to the improper design process, where workers have to work in an inappropriate location or a restricted area. The structure similarity of the node and the influence rate on X1 have been ranked third, which can control human errors better together with K2. In the state of design failure, the workers need to focus most of their effort to overcome the uncomfortable workspace that distract their attention. When designing construction operations, the actual situation of the site should have checked in advance for planning and arranging necessary construction machineries or manual operations.

Regarding the intermediate node, D4 (inattention) means workers neglect a signal or an event as per their carelessness. Although the inattention does not exist in wrong sequence, it is necessary to control the inattention in wrong action and wrong target based on its structure similarity (0.62), which the influence rate on X1 is the highest. Due to a large volume of materials and machineries, the construction site is often chaotic. It will greatly distract the attention of workers. Therefore, allocation of more warning signs, warning lights, safety education and training are necessary to control the inattention issues. 


\section{Discussions and Conclusions}

The research has identified on influence factors and effective control variables for human errors based on the proposed topological structure. The integration of CREAM and Bayesian structural learning have incorporated the expert knowledge and actual industry data for the final causal model of human errors, which could be the most comprehensive modeling approach. The reliability, similarity and sensitivity of the topology were conducted to (a) analyze the similarities and differences between different models and (b) rank the importance of nodes. These analyses have extended the depth and breadth of this research. Besides, the research also contributes useful and practical implications. We have analyzed the similarity of nodes in the topology and the influence rate of nodes on human errors based on the sensitive analysis. These results render new and practical ideas for safety management, particularly to control and reduce the occurrence of human errors.

According to the analysis of the nodes in topology, the most importance of the property of the nodes between each other are influencing rate from sensitivity analysis and similarity analysis. The similarity of nodes will reflect nodes' mechanism in different topologies, the higher the similarity, the more generally affect all the models by controlling the specific node. Moreover, the influence rate of the node will reflect the quantitative change of probability of human error after controlling this node, the higher the influence rate, the higher the sensitivity of human error to the node. Therefore, in order to achieve more efficient safety management, we should focus on the nodes that have both high similarity and high influence rate on human errors. By comparing the results between Table 3 and Table 5, the ranking order of nodes is rather similar. In other words, the nodes of high similarity consider as the key factor in controlling human errors. Therefore, risks can be effectively avoided by controlling these antecedent errors and subsequently, it can reduce the 
occurrence of the three types of human errors.

Certain limitations need to be considered in the research. Due to the node variables (risks) are coexisted at the same time based on the CREAM approach, the factors that change over time are unable to capture in the theoretical models. Hence, future works could take the aspect of time into account, especially to investigate the mechanism for a long time. Furthermore, human errors are a period of wrong behaviors for one person, so it is possible to integrate the three models into a synthesized model. Besides, there are many restrictions when implementing the strategy to improve multiple factors at the same time in on-site management. When the management does not achieve the intended target, the invested resources will be in vain. The future research should focus on the joint management of the most effective nodes in controlling human errors. Nevertheless, the findings provide new theoretical insights and practical ideas into the analytical approach in safety management by consolidating the influential mechanics in addressing human errors through the case study on the elevator installation. The research has also delved the details of the key factors and put forward the corresponding management measures to avoid the occurrence of key antecedents and human errors.

\section{References}

Abdelhamid, T. S., \& Everett, J. G. (2000). "Identifying root causes of construction accidents." Journal of construction engineering and management, Vol. 126, No. 1, pp. 52-60, DOI: 10.1061/(ASCE)07339364(2000)126:1(52).

Akyuz, E., \& Celik, M. (2015). "Application of CREAM human reliability model to cargo loading process of LPG tankers." Journal of Loss Prevention in the Process Industries, Vol. 34, pp. 39-48, DOI: 10.1016/j.jlp.2015.01.019. 
Bureau of Labor Statistics (2015). U.S. Department of Labor. Fatal Occupational Injuries by Event or Exposure. Available from: http://www.bls.gov/iif/oshcfoi1.htm\#

Fang, D., Zhao, C., \& Zhang, M. (2016). "A Cognitive Model of Construction Workers' Unsafe Behaviors." Journal of construction engineering and management, Vol. 142, No. 9, pp. 04016039, DOI: 10.1061/(ASCE)CO.1943-7862.0001118.

García-Herrero, S., Mariscal, M. A., García-Rodríguez, J., \& Ritzel, D. O. (2012). "Working conditions, psychological/physical symptoms and occupational accidents. Bayesian network models." Safety science, Vol. 50, No. 9, pp. 1760-1774, DOI: 10.1016/j.ssci.2012.04.005.

Griffin, M. A., \& Neal, A. (2000). "Perceptions of Safety at Work: A Framework for Linking Safety Climate to Safety Performance, Knowledge, and Motivatioon." Journal of Occupational Health Psychology, Vol. 5, No. 3, pp. 347-358, DOI: 10.1037/1076-8998.5.3.347.

Hayhurst. (1959). "Industrial Accident Prevention. A Scientific Approach." Industrial \& Labor Relations Review, Vol. 4, No. 4, pp. 609, DOI: 10.2105/AJPH.22.1.119-b.

Hollnagel, E. (1998). "Cognitive Reliability and Error Analysis Method (CREAM)", Elsevier Science.

. Li, D. Q., Tang, X. S., Zhou, C. B., \& Phoon, K. K. (2015). "Characterization of uncertainty in probabilistic model using bootstrap method and its application to reliability of piles." Applied Mathematical Modelling, Vol. 39, No. 17, pp. 5310-5326, DOI: 10.1016/j.apm.2015.03.027.

Li, P. C., Chen, G. H., Dai, L. C., \& Zhang, L. (2012). "A fuzzy Bayesian network approach to improve the quantification of organizational influences in HRA frameworks." Safety science, Vol. 50, No. 7, pp. 1569-1583, DOI: 10.1016/j.ssci.2012.03.017.

Liao, B, Gao, H., Liu, M \& Yang, Q. (2012). "Method of human reliability analysis on school bus driver based on CREAM." Journal of Safety Science and Technology, Vol. 8, No. 11, pp. 145-150. 
Liao, P. C., Luo, X., Wang, T., \& Su, Y. (2016). "The Mechanism of how Design Failures cause Unsafe Behavior: The Cognitive Reliability and Error Analysis Method (CREAM)." Procedia Engineering, Vol. 145, pp. 715-722, DOI: 10.1016/j.proeng.2016.04.088.

Melnik, S., Garcia-Molina, H., \& Rahm, E. (2002). "Similarity Flooding: A Versatile Graph Matching Algorithm and Its Application to Schema Matching." International Conference on Data Engineering, 2002. Proceedings, pp. 117-128, DOI: 10.1109/ICDE.2002.994702.Mohammadfam, I., Ghasemi, F., Kalatpour, O., \&

Mitropoulos, P. T. (2009). "The role of production and teamwork practices in construction safety: a cognitive model and an empirical case study." Journal of Safety Research, Vol. 40, No.4, pp. 265-275, DOI: 10.1016/j.jsr.2009.05.002.

Moghimbeigi, A. (2017). "Constructing a Bayesian network model for improving safety behavior of employees at workplaces." Applied Ergonomics, Vol. 58, pp. 35-47, DOI: 10.1016/j.apergo.2016.05.006.

Neal, A., Griffin, M. A., \& Hart, P. M. (2000). "The impact of organizational climate on safety climate and individual behavior." Safety science, Vol. 34, No. 1-3, pp. 99-109, DOI: 10.1016/S0925-7535(00)00008-4.

Othman, M. M., \& Musirin, I. (2011). "A novel approach to determine transmission reliability margin using parametric bootstrap technique." International Journal of Electrical Power \& Energy Systems, Vol. 33, No. 10, pp. 1666-1674, DOI: 10.1016/j.ijepes.2011.08.003.

Pierce, B. (2016). "How rare are large, multiple-fatality work-related incidents?" Accident Analysis \& Prevention, Vol. 96, pp. 88-100, DOI: 10.1016/j.aap.2016.07.014.

Rodrigues, A. B., \& Silva, M. D. G. D. (2013). "Confidence Intervals Estimation for Reliability Data of Power Distribution Equipments Using Bootstrap." IEEE Transactions on Power Systems, Vol. 28, No. 3, pp. 3283-3291, DOI: 10.1109/TPWRS.2012.2235471.

Skalle, P., Aamodt, A., \& Laumann, K. (2014). "Integrating human related errors with technical errors to determine 
causes behind offshore accidents." Safety science, Vol. 63, No. 3, pp. 179-190, DOI: 10.1016/j.ssci.2013.11.009.

Streukens, S., \& Leroi-Werelds, S. (2016). "Bootstrapping and PLS-SEM: A step-by-step guide to get more out of your bootstrap results." European Management Journal, Vol. 34, No. 6, pp. 618-632, DOI: 10.1016/j.emj.2016.06.003.

Shen Z, Wang, Y, \& Gao, J. (2005). "Consequent-antecedent retrospective table for human errors." Journal of Tsinghua University (Science and Technology), Vol. 45, No. 6, pp. 799-802, DOI: 10.16511/j.cnki.qhdxxb.2005.06.021.

Wang, Y. \& Shen, Z. (2005). "CREAM-—A Second-Gneration Human Reliability Analysis Method", Industrial Engineering and Management, No. 3, pp. 17-21

Xie, Y., \& Zhu, Y. (2008). "Bootstrap Methods: Developments and Frontiers." Statistics \&Information Forum, Vol. 23, No. 2, pp. 90-96. 